\title{
Efficacy of phosphocreatine pre-administration on XIAP and Smac in ischemic penumbra of rats with focal cerebral ischemia reperfusion injury ${ }^{1}$
}

\author{
Wei Wang', Qi Wang', Wanyou Yu', Lianhua Chen", Zhong Li"I'
}

'MD, Department of Anesthesiology, the Jiangning Hospital Affiliated, Nanjing Medical University, China. Acquisition and analysis of data, manuscript writing.

"MD, Department of Anesthesiology, the First People's Hospital of Shanghai Affiliated, Nanjing Medical University, Shanghai, China. Analysis and interpretation of data.

I'IPhD, Professor, Key Laboratory of Modern Toxicology (Ministry of Education), School of Public Health, Nanjing Medical University, China. Conception and design of the study, critical revision, final approval the manuscript.

\begin{abstract}
Purpose: To observe the efficacy of phosphocreatine pre-administration (PCr-PA) on X-linked inhibitor of apoptosis protein (XIAP), the second mitochondia-derived activator of caspase (Smac) and apoptosis in the ischemic penumbra of rats with focal cerebral ischemiareperfusion injury (CIRI).

Methods: A total of 60 healthy male Sprague Dawley (SD) rats were randomly divided into three groups ( $n=20)$ : group $A$ (the sham operation group), group $B$ [intraperitoneally injected with $20 \mathrm{mg} / \mathrm{kg}(10 \mathrm{mg} / \mathrm{ml})$ of saline before preparing the ischemia-reperfusion (IR) model], and group C [intraperitoneally injected with $20 \mathrm{mg} / \mathrm{kg}(10 \mathrm{mg} / \mathrm{ml})$ of PCr immediately before preparing the IR model]. After $24 \mathrm{~h}$ for reperfusion, the neurological function was evaluated and the tissue was sampled to detect expression of XIAP, Smac and caspase-3 positive cells in the ischemic penumbra so as to observe the apoptosis.

Results: Compared with group B, neurological deficit scores, numbers of apoptotic cells, expression of Smac,caspase- 9 and the numbers of Caspase-3 positive cells were decreased while expression of XIAP were increased in the ischemic penumbra of group C.

Conclusions: Phosphocreatine pre-administration may elicit neuroprotective effects in the brain by increasing expression of X-linked inhibitor of apoptosis protein, reducing expression of second mitochondia-derived activator of caspase, and inhibiting the apoptosis in the ischemic penumbra.
\end{abstract}

Key words: Phosphocreatine. Reperfusion Injury. X-Linked Inhibitor of Apoptosis Protein. Caspases. Rats. 


\section{- Introduction}

The key to treating ischemic stroke lies in recanalizing partial vessels or thrombolytic therapy, which can rescue or reduce neuronal apoptosis in the ischemic penumbra in a timely manner ${ }^{1,2}$. The ischemic cerebral tissue outside and surrounding the focal central infarction area is called the ischemic penumbra. Since it is a reverse injury area, its function can be restored if treated timely and effectively ${ }^{3,4}$. Studies have shown that CIRI is associated with metabolic disorders, energy failure, oxygen free radicals, calcium overload, NO, inflammatory response, excitatory amino acids, cerebral edema, or apoptosis ${ }^{5-7}$. Cell apoptosis after cerebral ischemia-reperfusion injury relies on endogenous death receptor pathway and mitochondrial apoptosis pathway. In mitochondrial apoptosis pathway, apoptosispromoting protein is released from mitochondrial intermembrane space and redistributed into the cytoplasm, leading to caspase activation and cell apoptosis ${ }^{8}$. Smac, a type of apoptosis-promoting protein,can antagonize caspase-inhibiting XIAP, accelerating the process of cell apoptosis ${ }^{9}$. Studies have showed that $\mathrm{PCr}$ has effects of brain protection, but the specific mechanism is not clear yet ${ }^{10,11}$. The current study investigated the efficacy of PCr-PA on XIAP, Smac and apoptosis in the ischemic penumbra of rats a focal CIRI, with the aim of exploring the possible mechanism by which $\mathrm{PCr}$ can protect the brain.

\section{- Methods}

This study was carried out in strict accordance with the recommendations in the Guide for the Care and Use of Laboratory Animals of the National Institutes of Health. The animal use protocol has been reviewed and approved by the Institutional Animal Care and Use Committee (IACUC) of Nanjing Medical
University.

A total of 60 male SD rats, weighing 280-320 g, aged 9-10 weeks, were provided by Nanjing Medical University. They were randomly divided into three groups $(n=20)$ as follows: group A of sham operation; group $B$, which were intraperitoneally injected with $20 \mathrm{mg} / \mathrm{kg}(10 \mathrm{mg} / \mathrm{ml})$ of saline immediately before preparing the IR model; and group $\mathrm{C}$, which were intraperitoneally injected with $\mathrm{PCr}$ (Haikou Qili Pharmaceutical Co., Ltd., Hainan, China) at a dose of $20 \mathrm{mg} / \mathrm{kg}(10 \mathrm{mg} / \mathrm{ml})$ immediately before preparing the IR model.

The rats were fasted for $12 \mathrm{~h}$ (but were free to drink water) before surgery. To prepare the CIRI model, each rat was then prepared to undergo one of the modified modeling method $^{12}$. The rats were anesthetized by an intraperitoneal injection of $2 \%$ pentobarbital sodium $(50 \mathrm{mg} / \mathrm{kg})$ and retained spontaneous breathing. A median neck incision was then made to expose the right common carotid artery, internal carotid artery, and external carotid artery. Thereafter, an incision was made under the branch of the common carotid artery, and one round nylon line (3.0, Ethicon, USA), which was approximately $17.5 \mathrm{~mm}$ in length (starting from the branch of the internal and external carotid arteries), was placed into the internal carotid artery until a slight resistance was felt. The internal carotid artery was then ligated for $2 \mathrm{~h}$, and the thread was then pulled out to allow for a 24-h reperfusion. Rats in group $S$ underwent the same steps as above, except that the insertion depth of the thread was less than $10 \mathrm{~mm}$. One multifunction monitor with an anal temperature probe was used during surgery to monitor the body temperature, which was maintained at $37.0-37.5^{\circ} \mathrm{C}$ using one roasted lamp. The modeling was thought to be successful when the Homer sign appeared in the right eye, the left forelimb abducted when the tail was lifted, and the rat crawled to the left or turned 
counterclockwise. The rats in group B and C that did not meet the above performance criteria were excluded from the current study. Please note however, that all animals survived and none were excluded in each group.

\section{Neurological function assessment}

After the reperfusion, the neurological deficit was marked using a method described previously ${ }^{12}$. The following scale was used: 0 point: no neurological deficit; 1 point: the contralateral forelimb cannot be completely stretched; 2 points: turned around to the hemiplegic side while walking; 3 points: dumped to the hemiplegic side; and 4 points: cannot walk on its own and consciousness is depressed.

\section{Definition of infarcted core area and ischemic penumbra}

The infarcted core area and ischemic penumbra were defined using the same microscope, as previously described ${ }^{13}$.The tissue was then stained with hematoxylin and eosin (HE).

\section{Histopathological observation}

Brain tissue from a total of 10 rats, which were randomly sampled from each group and perfused using 4\% paraformaldehyde, were obtained. The coronal brain tissue was then sampled, fixed, embedded with paraffin, and prepared as 6-mm slices for HE staining, using the optic chiasm as a reference point. The neuronal morphology, necrosis, and tissue structure were then observed using light microscopy.

\section{Determination of apoptosis}

The samples were first embedded in paraffin embedding according to the manufacturer instructions of the deoxynucleotidyl transferase dUTP nick end labeling (TUNEL) kit (Precinorm, Germany), before undergoing diaminobenzidine (DAB) coloration and $\mathrm{HE}$ re-staining. The results were determined as follows: the nuclei of the apoptotic cells appeared buffy, while the nuclei of normal cells were blue. The positive cells in five, randomly selected high-magnification fields of the same slide ( $\times 400$ times) were counted. The average number of positive cells were then calculated.

\section{Expression of XIAP and Smac}

A random sample of 10 rats from each group, were sacrificed, and their brain tissue was removed and underwent a 5-min immediate quick freezing at $-20^{\circ} \mathrm{C}$. Using a previously described method ${ }^{13}$, the coronal segments were then sliced at sites, which were $3 \mathrm{~mm}$ and $9 \mathrm{~mm}$ away from the frontal lobe front. The brain tissue ( $6 \mathrm{~mm}$ in thickness) within the above segment was then cut open along the line $2 \mathrm{~mm}$ away from the sagittal suture. The median structures of these two hemispheres, which are supplied with blood by the anterior cerebral artery, were then removed. The remaining left and right sides were then cut sagittally along the line, which was $2 \mathrm{~mm}$ away and at a $30^{\circ}$ oblique to the sagittal section. The lateral cortex and the medial cortex were the infarcted core area and ischemic penumbra, respectively.Tissues of the ischemic penumbra were taken out. Sampling and paraffin embedding were made with freezing at $-80 \stackrel{\circ}{ } \mathrm{C}$. The tissue homogenate was taken to extract the total protein, then the protein was quantified by bicinchoninic acid (BCA). A dose of $70 \mu \mathrm{g}$ protein was transferred to polyvinylidence fluoride (PVDF) membrane after polyacrylamide gel electrophoresis, and closed by $5 \%$ skim milk for $1 \mathrm{~h}$ at room temperature. The PVDF membrane was marked and segmented by standard pre-stained protein molecular weight, 
then incubated with anti-XIAP,Smac (santa cruz, USA), aspase- 9 (CST, USA) and anti- $\beta$ actin(Immunoway, USA) at 4 으 overnight, and then with horseradish peroxidase(HRP)-antiantibody (Jackson ImmunoResearch,USA) for $2 \mathrm{~h}$ at room temperature. After luminescence, development, fixation and scanning of $E C L$, the image was analyzed by software of Image.Pro. Plus6.0 (Media Cybemetics USA). The protein expression level was quantified by the ratio of the gray value of the protein stripe to the corresponding $\beta$-actin.

\section{Determination of caspase-3 positive cells}

After paraffin section and hydration, detection of SP and coloration of DAB, the cytoplasm of positive cells became brownish yellow. Each slice were selected three fields(x200), then the positive cells were counted in the ischemic penumbra.

\section{Statistical analysis}

All statistical analyses were performed with SPSS for Windows (version 16.0; SPSS Inc. Chicago, IL, USA). Measurement data were expressed as mean \pm standard deviation ( $x$ $\pm s)$. The differences among different regions and among different groups were analyzed using a one-way analysis of variance (ANOVA). Statistical significance was set at $P<0.05$.

\section{Results}

Structure and cell morphology distribution in the infarct brain tissues

Cell morphology of brain tissues was normal in group A. The ischemic penumbra, area between the infarcted foci and normal regions in group $B$, demonstrated its special cell morphology, indicating that the infarcted core area mainly covered the striatum, parietal cortex, and partial frontal cortex. The infarcted core area in group $C$ was smaller than that in group $B$, mainly covering the striatum and partial parietal cortex.

\section{Neurological function scoring}

Compared with group $A$, the neurological deficit scores of group $B$ and $C$ were increased $(P<0.01)$. The neurological deficit score of group $C$ was lower than that in group $B(P<0.05)$ (Table 1$)$.

Table 1 - Comparison of neurological deficit scores among different groups.

\begin{tabular}{ccc} 
Group A & Group B & Group C \\
\hline 0 & $2.6 \pm 0.5^{\mathrm{a}}$ & $1.4 \pm 0.6^{\mathrm{ab}}$ \\
\hline
\end{tabular}

All values are the mean \pm standard deviation. $n=20$ per group. The neurological deficit was described after the reperfusion. ${ }^{a} P<0.01$ vs. group $A ;{ }^{b} P<0.05$ vs. group $B$.

\section{Comparison of apoptosis}

Compared with group $A$, the numbers of apoptotic cells in the infarcted core area and ischemic penumbra of group $B$ and $C$ increased $(P<0.01)$. Compared with group $B$, the numbers of apoptotic cells in the infarcted core area and ischemic penumbra of group $C$ decreased ( $P$ $<0.05)$.Compared with the infarcted core area, the numbers of apoptotic cells in the ischemic penumbra of group $B$ and $C$ increased $(P<0.05)$ (Table 2).

Table 2 - Comparison of apoptotic cells among different groups.

\begin{tabular}{ccc} 
Group & $\begin{array}{c}\text { Infarcted core } \\
\text { area }\end{array}$ & $\begin{array}{c}\text { Ischemic } \\
\text { penumbra }\end{array}$ \\
\hline A & $4.1 \pm 0.9$ & $3.9 \pm 0.7$ \\
B & $15.9 \pm 1.6^{\mathrm{a}}$ & $46.9 \pm 3.3^{\mathrm{ac}}$ \\
C & $15.5 \pm 1.7^{\mathrm{a}}$ & $25.6 \pm 2.7^{\mathrm{abc}}$ \\
\hline
\end{tabular}

All values are the mean \pm standard deviation. $n=10$ per group. The infarcted core area and ischemic penumbra were followed manual instructions of TUNEL kit, then colored by DAB and restrained by hematoxylin. ${ }^{a} P<0.01$ vs. group $A ;{ }^{b} P<0.05$ vs. group $B ;{ }^{c} P<0.05$ vs. the infarcted core area.TUNEL, deoxynucleotidyl transferase dUTP nick end labeling; DAB, diaminobenzidine. 


\section{Expression of XIAP and Smac}

Compared with group A, XIAP expression decreased and Smac expression increased significantly in group $B$ and $C(P$
$<0.01) 24 \mathrm{~h}$ after reperfusion. Compared with group $B, X I A P$ expression increased and Smac expression decreased in group $C(P<0.05)$ (Figure 1).
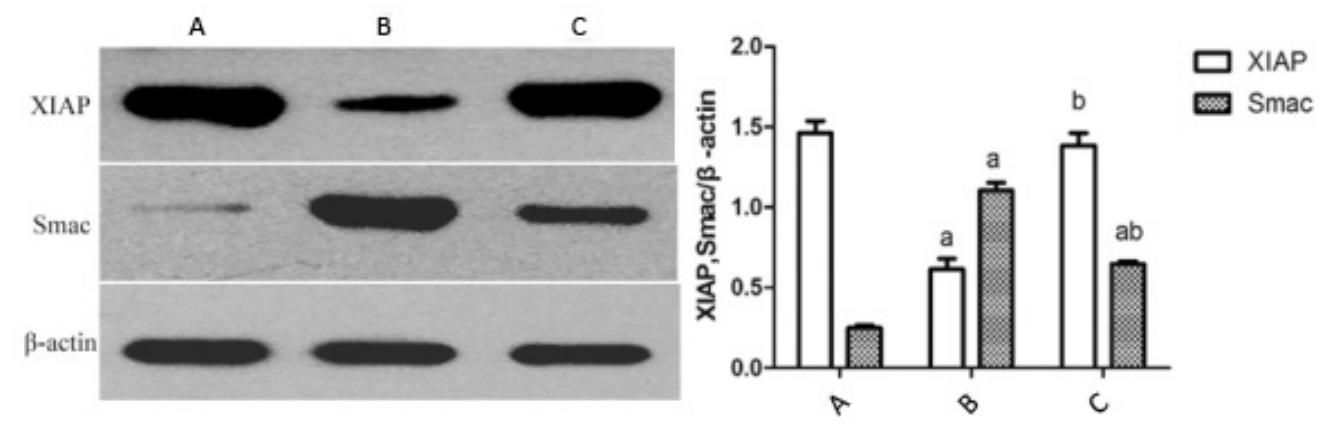

Figure 1 - The expression of XIAP and Smac proteins in ischemic penumbra after $24 \mathrm{~h}$ of ischemia reperfusion in the three groups. The protein expression level of XIAP and Smac was quantified by the ratio of the gray value of the protein stripe to the corresponding $\beta$-actin. ${ }^{a} P<0.05$ vs. group $A ;{ }^{b} P<0.05$ vs. group $B$. XIAP, $x$-linked inhibitor of apoptosis protein; Smac, the second mitochondia-derived activator of caspase.

\section{Expression of caspase-9}

Compared with group A, caspase-9 expressions increased significantly in group
$B$ and $C(P<0.05) 24 \mathrm{~h}$ after reperfusion. Compared with group $B$, caspase-9 expression decreased in group $C(P<0.05)$ (Figure 2$)$.
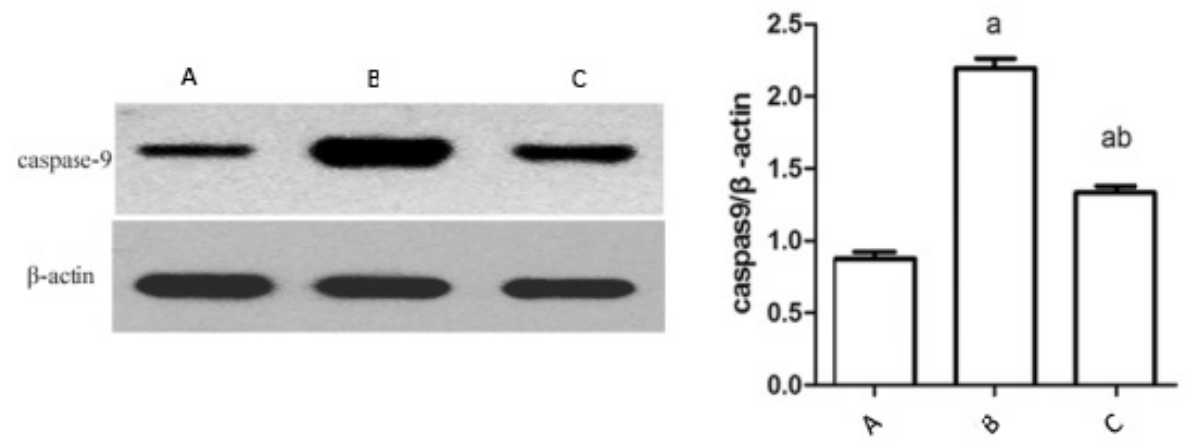

Figure 2 - The expression of caspase- 9 proteins in ischemic penumbra after $24 \mathrm{~h}$ of ischemia-reperfusion in the three groups. The protein expression level of caspase- 9 was quantified by the ratio of the gray value of the protein stripe to the corresponding $\beta$-actin. ${ }^{a} P<0.05$ vs. group $A ;{ }^{b} P<0.05$ vs. group $B$.

Expression of caspase-3 positive cells

Compared with group $A$,the number of Caspase-3 positive cells in ischemic penumbra increased in group $B$ and $C(P<0.05)$. Compared with group $B$, the number of Caspase- 3 positive cells decreased in gruop C $(P<0.05)$ (Table 3 and Figure 3). 
Table 3 - Comparison of caspase-3 positive cells among different groups.

\begin{tabular}{ccc}
\hline Group A & Group B & Group C \\
\hline$(97.3 \pm 6.3)$ & $(152.1 \pm 9.2)^{\mathrm{a}}$ & $(115.1 \pm 6.4)^{\mathrm{ab}}$ \\
\hline
\end{tabular}

All values are the mean \pm standard deviation. $n=10$ per group. Each slice were selected three fields ( $x 200$ ), then the positive cells were counted in the ischemic penumbra. ${ }^{a} P<0.01 \mathrm{vs}$. group $A ;{ }^{b} P<0.05$ vs. group $B$.

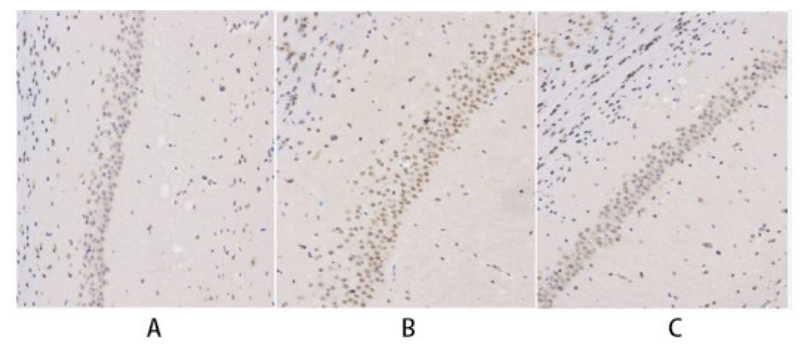

Figure 3 - The expression of caspase-3 cells in ischemic penumbra after $24 \mathrm{~h}$ of ischemia reperfusion in the three groups $D A B, \times 200)$. After paraffin section and hydration, detection of $S P$ and coloration of DAB, the cytoplasm of positive cells became brownish yellow. A random image of each group was selected above. SP,streptavidinperosidase; $\mathrm{DAB}$, diaminobenzidine.

\section{Discussion}

When preparing the rat model of CIRI, given its good stability, ease (since there is no need of a craniotomy), and low trauma, the suture-embolus method can be used to accurately control the ischemic and reperfusion time, with a high success rate and low mortality ${ }^{14}$. In the current study, the IR rats exhibited significant infarction signs, increased neurological deficit scores, and significant brain injury. Since the main site of lesion was adjacent to the optic chiasm, sampling sites were set $3 \mathrm{~mm}$ before and after the optic chiasm.

In the present study, the infarcted foci in the IR rats mainly involved the striatum, parietal cortex, and partial frontal cortex, which was almost consistent with a previously study conducted by Memezawa ${ }^{15}$. The results showed that although $\mathrm{PCr}$ can increase the expression of XIAP and reduce the expression of Smac in infarcted core region to a certain extent, the striatum was the tissue firstly involved in the infarcted core area; so PCr could not reverse serious injury of the striatum and could only reduce the injury in partial parietal cortex and frontal cortex. According to the positioning method of the cerebral ischemic penumbra in rats $^{13}$ and the HE staining results, these areas made up the ischemic penumbra. In addition, TUNEL staining results showed that $\mathrm{PCr}$ could not reduce the number of apoptotic cells in the infarcted core region. These results suggested that $\mathrm{PCr}$ mainly reversed the injury in the ischemic penumbra, not the infarcted core area. Therefore, we compared the expression of XIAP, Sam, caspase- 3 and caspase- 9 in the ischemic penumbra tissue of rats.

Regulated by the caspase family, apoptosis is one of the main mechanisms governing cell death after cerebral ischemiareperfusion injury. Once activated by caspase, it triggers an irreversible cascade reaction ${ }^{16}$.The inhibitor of apoptosis proteins(IAPs) family may regulate the activation of caspase, including c-IAPI, c-IAP2, XIAP and Survivin. XIAP, directly associated with Caspase-3, caspase-7 and caspase- 9 , is the strongest caspase inhibitor ${ }^{17}$. Saito et al. ${ }^{18}$ believe that activation of the XIAP pathway occurs prior to the activation of caspase cascade, and the interaction between XIAP and Smac and caspase is critical for neuronal cell death after cerebral ischemia. Our study showed that the expression of XIAP decreased and the expression of caspase- 9 and caspase-3 increased in ischemic penumbra of group B at $24 \mathrm{~h}$. However, the expression of XIAP increased and the expression of caspase- 9 and caspase-3 decreased in group $\mathrm{C}$. The activation of caspase- 9 or caspase- 3 after ischemia might 
increase the degradation of XIAP. PCr can promote the expression of XIAP (or inhibit its degradation), inhibit the activity of caspase- 9 and Caspase-3, and reduce apoptosis in the penumbra zone of cerebral ischemia, all of which contribute to its protection for the brain.

Smac, located in the intermembrane space, is one of the negative regulatory gene for XIAP. Under the stimulation of apoptosis, Smac in the cytoplasm directly combines with IAPs (especially XIAP), inhibiting their anti-apoptotic activity ${ }^{19}$. It has been found that expression inhibition of Smac is one of the key mechanisms in the protection against cerebral ischemic injury $^{20}$. Up-regulation of XIAP and downregulation of Smac improve the prognosis of cerebral ischemia ${ }^{21}$.This study showed that the expression of Smac in the ischemic penumbra region increased significantly $24 \mathrm{~h}$ after cerebral ischemia reperfusion in the group $B$, suggesting that Smac is involved in the death of cells after ischemia. The expression of Smac in group $C$ was significantly lower than that in group $B$, suggesting that $\mathrm{PCr}$ can alleviate the focal cerebral ischemia-reperfusion injury by reducing the expression of Smac in the ischemic penumbra.

\section{Conclusions}

Phosphocreatine pre-administration may elicit neuroprotective effects in the brain by increasing expression of $\mathrm{X}$-linked inhibitor of apoptosis protein, reducing expression of second mitochondia-derived activator of caspase, and inhibiting the apoptosis in the ischemic penumbra. Thus, it delayed the progression of the ischemic penumbra toward infarcted core area and reduced the volume of the infarct. Therefore, given its neuroprotective effects, we postulate that PCrPA can improve the prognosis of patients with cerebral ischemia reperfusion injury.

\section{References}

1 Hongyun $\mathrm{H}$, Tao G, Pengyue Z, Liqiang Y, Yihao D. Puerarin provides a neuroprotection against transient cerbral ischemia by attenuating autophagy at the ischemic penumbra in neurons but not in astrocytes. Neurosci Lett. 2017 Mar 16;643:45-51. doi: 10.1016/j.neulet.2017.02.009.

2 Mcleod D. Ischemic penumbra in retina endures: vascular neuropathology is reconciled. Neural Regen Res. 2016 May;11(5):737-9. doi: 10.4103/16735374.181367.

3 Astrup J, Symon L, Branston NM, Lassen NA. Cortical evoked potential and extracellular $\mathrm{K}^{+}$and $\mathrm{H}^{+}$at critical levels of brain ischemia. Stroke. 1977 Jan-Feb;8(1):51-7. PMID: 13521.

4 Catanese L, Tarsia J, Fisher M. Acute ischemic stroke therapy overview. Circ Res. 2017 Feb;120(3):541-58. doi: 10.1161/ CIRCRESAHA.116.309278.

5 Fouda AY, Pillai B, Dhandapani KM, Ergul A, Fagan SC. Role of interleukin-10 in the neuroprotective effect of the Angiotensin Type 2 Receptor agonist, compound 21,after ischemia/reperfusion injury. Eur J Pharmacol. 2017 Mar;799:128-34. doi: 10.1016/j.ejphar.2017.02.016.

6 Kim AY, Jeong $\mathrm{KH}$, Lee $\mathrm{JH}$, Kang $\mathrm{Y}$, Lee SH, Baik EJ. Glutamate dehydrogenase as a neuroprotective target against brain ischemia and reperfusion. Neuroscience. 2017 Jan;340:487-500. doi: 10.1016/j. neuroscience.2016.11.007.

7 Fakharnia F, Khodagholi F, Dargahi L, Ahmadiani A. Prevention of cyslopholin d-mediated MPTP opening using cyclosporine-a alleviates the elevation of necroptosis, autophagy and apoptosisrelated markers following global cerebral ischemia-reperfusion. J Mol Neurosci. 2017 Jan;61(1):52-60. doi: 10.1007/s12031-0160843-3.

8 Nagley P, Higgins GC, Atkin JD, Beart PM. Multifaceted deaths orchestrated by mitochondria in neurons. Biochim Biophys Acta. 2010 Jan;1802(1):167-85. doi: 10.1016/j.bbadis.2009.09.004.

$9 \mathrm{Li} J$, Li Y, Qin D, von Harsdorf R, Li P. Mitochondrial fission leads to Smac/DIABLO release quenched by ARC. Apoptosis. 2010 Oct;15(10):1187-96. doi: 10.1007/s10495010-0514-8. 
10 Balestrino $M$, Lensman $M$, Parodi M, Perasso L, Rebaudo R, Melani R, Polenov S, Cupello A. Role of creatine and phosphocreatine in neuronal protection from anoxic and ischemic damage. Amino Acids. 2002;23(13):221-9. doi: 10.1007/s00726-001-0133-3.

11 Lwata O, Lwata S, Bainbridge A, De Vita E, Matsuishi T, Cady EB, Robertson NJ. Supra-and sub-baseline phosphocreatine recovery in developing brain after transient hypoxia-ischaemia:relation to baseline energetics,insult severity and outcome. Brain. 2008 Aug;131(Pt 8):2220-6. doi: 10.1093/brain/awn150.

12 Longa EZ, Weinstein PR, Carlson S, Cummins R. Reversible middle cerebral artery occlusion without craniectomy in rats. Stroke. 1989 Jan;20(1):84-91. PMID: 2643202.

13 Ashawal S, Tone B, Tian HR, Cole DJ, Pearce WJ. Core and penumbral nitric oxide synthase activity during cerebral ischemia and reperfusion. Stroke. 1998 May;29 (5):1037-46. PMID: 9596255.

14 Hara T, Mies G, Hata R, Hossmann KA. Gene expressions after thrombolytic treatment of middle cerebral artery clot embolism in mice. Stroke. 2001 Aug;32(8):1912-9. PMID: 11486125.

15 Memezawa $H$, Smith ML, Siesjo BK. Penumbral tissues salvaged by reperfusion following middle cerebral artery occlusion in rats. Stroke. 1992 Apr;23(4):552-9. PMID: 1561688.
16 Nakka VP, Gusain A, Mehta SL, Raghubir R. Molecular mechanisms of apoptosis in cerebral ischemia: multiple neuroprotective opportunities. Mol Neurobiol. 2008 Feb;37(1):7-38. doi:10.1007/s12035-0078013-9.

$17 \mathrm{Su}$ BC, Mo FE. CCN1 enables Fas ligandinduced apoptosis in cardiomyoblast H9c2 cells by disrupting caspase inhibitor XIAP. Cell Signal. 2014 Jun;26(6):1326-34. doi: 10.1016/j.cellsig.2014.02.019.

18 Saito A, Hayashi T, Okuno S, Nishi T, Chan $\mathrm{PH}$. Oxidative stress is associated with XIAP and Smac/DIABLO signaling pathways in mouse brains after transient focal cerebral ischemia. Stroke. 2004 Jun;35(6):1443-8. doi: 10.1161/01.STR.0000128416.28778.7a.

19 Nijboer $\mathrm{CH}$, Heijnen CJ, van der Kooij MA, Zijlstra J, van Velthoven CT, Culmsee C, van Bel $\mathrm{F}$, Hagberg H, Kavelaars A. Targeting the p53 pathway to protect the neonatal ischemic brain. Ann Neurol. 2011 Aug;70(2):255-64. doi: 10.1002/ana.22413.

$20 \mathrm{Li} \mathrm{H}$, Wang D. Mild hypothermia improves ischemic brain function via attenuating neuronal apoptosis. Brain Res. 2011 Jan;1368:59-64. doi: 10.1016/j. brainres.2010.10.073.

21 Attaran-Bandarabadi F, Abhari BA, Neishabouri SH, Davoodi J. Integrity of XIAP is essential for effective activity recovery of apoptosome and its downstream caspases by Smac/Diablo. Int J Biol Macromol. 2017 Aug;101:283-9. doi: 10.1016/j. ijbiomac.2017.03.088.

\section{Correspondence:}

Zhong Li

Key Laboratory of Modern Toxicology

(Ministry of Education)

School of Public Health, Nanjing Medical

University No. 818 East Tianyuan Road,

Jiangning District China

Phone: +86 2586868451

Fax: +862586868451

Iz-ny@njmu.edu.cn

Received: Oct 26, 2017

Review: Dec 27, 2017

Accepted: Jan 29, 2018
Conflict of interest: none

Financial source: none
${ }^{1}$ Research performed at Central Laboratory, Department of Anesthesiology, Jiangning Hospital Affiliated, Nanjing Medical University, China. 